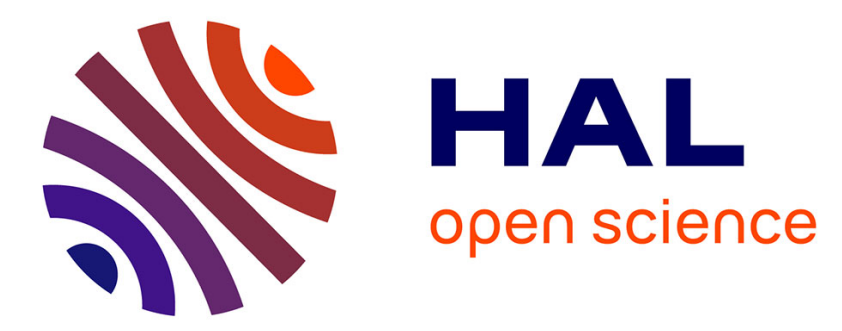

\title{
Towards WSN-aided Navigation for Vehicles in Smart Cities: An Application Case Study.
}

\author{
Roudy Dagher, Nathalie Mitton, Ibrahim Amadou
}

\section{To cite this version:}

Roudy Dagher, Nathalie Mitton, Ibrahim Amadou. Towards WSN-aided Navigation for Vehicles in Smart Cities: An Application Case Study.. 1st International IEEE Percom Workshop on Pervasive Systems for Smart Cities (PerCity 2014), Mar 2014, Budapest, Hungary. hal-00923611

\section{HAL Id: hal-00923611 \\ https://hal.inria.fr/hal-00923611}

Submitted on 5 May 2014

HAL is a multi-disciplinary open access archive for the deposit and dissemination of scientific research documents, whether they are published or not. The documents may come from teaching and research institutions in France or abroad, or from public or private research centers.
L'archive ouverte pluridisciplinaire HAL, est destinée au dépôt et à la diffusion de documents scientifiques de niveau recherche, publiés ou non, émanant des établissements d'enseignement et de recherche français ou étrangers, des laboratoires publics ou privés. 


\title{
Towards WSN-aided Navigation for Vehicles in Smart Cities : An Application Case Study
}

\author{
Roudy Dagher*†, Nathalie Mitton*, Ibrahim Amadou* \\ *Inria Lille - Nord Europe, FUN research group. \\ ${ }^{\dagger}$ Etineo company, R\&D Department. \\ Email: roudy.dagher@inria.fr,nathalie.mitton@inria.fr,ibrahim.amadou@inria.fr
}

\begin{abstract}
With the emergence of Smart City concept, Wireless Sensor Networks (WSNs) become one of the key technologies for instrumenting the city, and thus providing its inhabitants with various services meant to improve their daily life. One of the identified applications is Smart Street Lightning, where the lamps are in a mesh network for remote control and maintenance purposes. This paper proposes Ubiquitous Navigation System (UNS), a WSN-based navigation system, which takes benefit from the Smart Street Lightning system to provide a local navigation service. The positioning part of the system uses Angle of Arrival (AoA) measurements to estimate the vehicle position on the map. Based on a realistic network scenario, extracted from a city map using Google Maps, we study the performance of triangulation using AoA in a smart urban environment that exhibits topology related constraints. Simulations results show that such constraints lead to particular spatial distribution of the anchor nodes that affects both positioning accuracy and beacon packets reception rate. We also propose and evaluate the use of the network communication range as a technique to mitigate the effect of geometric dilution of precision (GDOP). The simulation results show that this technique successfully detected GDOPaffected positions and thus significantly enhanced the positioning accuracy.
\end{abstract}

\section{INTRODUCTION}

Nowadays, information and communication technologies (ICT) have become an essential part of urban development as a response to challenges regarding infrastructure and public services availability [1]. In this context, the concept of smart cities has attracted significant research interest, where the driving idea is to connect the growing number of population with the necessary services for their daily life.

With recent advances in the development of Wireless sensor networks (WSNs), such technology is envisioned to play a key role in instrumenting and interconnecting such smart urban environments [2]. Sensor nodes are typically equipped with processing, sensing, power management and communication capabilities. They are able to communicate in a wireless adhoc fashion over short radio communication range [3]. The resulting smart city will have a more efficient resource management and a better quality of life for the citizens by means of applications such as: Smart Parking, Traffic Management, Noise and Pollution Monitoring, Smart Lightning, etc.

In this work, we focus on the smart street lightning systems, where each lamp is equipped with a sensor node that is meant for both remote controlling purposes and predictive maintenance. Such systems already exist in the literature. For instance, in [4] authors propose a control network for a LED street lighting system, and implement a geographic routing strategy. In [5] authors develop a street lighting management system that uses a WSN for interconnecting the lamps.

Moreover, WSN is one of the key technologies that have been used for indoor localization [6]. Knowing the node's location enables a myriad of location-based applications such as object tracking, environment monitoring, intrusion detection, and habitat monitoring [7]. Location estimation also supports core network services such as: routing, topology control, coverage, boundary detection and clustering [6].

From this perspective, our proposal is to make use of the already deployed WSN infrastructure for smart street lightning to provide a GPS-like service to the vehicles in the city. This WSN-aided navigation becomes important especially in cluttered and urban environments or undergrounds where GPS reception fails, thus providing a continuous smart navigation service to the user. It is a cost effective solution, as no modifications are required on the existing smart street lightning infrastructure. The main motivation of our work is the density of the lamps in the city and the highway, and their interesting Line of Sight (LOS) conditions with respect to the vehicle. This offers numerous potential anchor devices, that can be used as reference nodes for localization. By opportunistically discovering at least two lamps in the vicinity of a car, the position of the vehicle can be estimated by means of Angle of Arrival (AoA) based triangulation.

The goal of this paper is to study the performance of triangulation using AoA in a realistic smart urban environment that exhibits topology related constraints. Such constraints lead to particular spatial distribution of the anchor nodes that affects both positioning accuracy and beacon packets reception rate.

\section{A. Related work}

The triangulation based positioning problems have been extensively studied in the context of $\mathrm{WSN}^{1}$ [7] [8] [9] and mobile robot navigation [10] [11] [12].

At the best of our knowledge, the use of the smart street lightning for localization purposes is novel, especially in the context of smart cities. The particularity of our study is in the network model: realistic network topology and medium access control influence on the performance of positioning algorithm. Unlike literature studies, we use geographic data

\footnotetext{
${ }^{1}$ And more generally in Ad Hoc networks
} 
from real world as input for the simulation. In addition, as network topology is extracted from the city map, we have no control on the anchors placement. We thus propose to mitigate the effect of dilution of precision by filtering against the communication range of the network. Conducted simulations show that the spatial distribution of the anchor nodes affects both positioning accuracy under the effect of the geometric dilution of precision) and beacon packets reception rate due to collisions on beacon packets. Results also show that the proposed GDOP mitigation technique successfully detects GDOP-affected positions and significantly enhances the positioning accuracy.

\section{B. Paper Organization}

The remainder of the paper is organized as follows. Section II draws the general scheme in which we propose AoA positioning for navigating in smart environments. Section III discusses the basic angle-based triangulation and its sensitivity to measurement noise, while Section IV describes the assumptions regarding the network. We present our proposed approach for the position estimation of the vehicle and its implementation issues in Section VI. In Section VII we discuss the simulation results. Finally, Section VIII concludes the paper.

\section{Ubiquitous NAVIGATION SYSTEM (UNS)}

In order to implement vehicular navigation in a smart city environment, we propose a hybrid strategy [13].

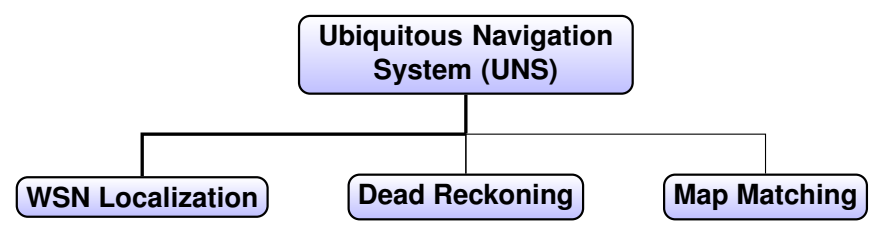

Fig. 1. Main components for smart navigation

The core function is position estimation by using rangebased measurements to the fixed nodes in the vicinity of the mobile node (WSN Localization). The Dead Reckoning function, based on a strapdown Inertial Navigation System (INS) is accurate over short period of times, and requires an initial position and heading estimate. Hence, it can be reset by the WSN Localization function upon every successful position estimate. Finally, the Map Matching function is used in a refinement phase, where the map knowledge is used to mitigate position estimation errors.

Withing this framework, in this paper, we focus on the WSN-based position estimation process. By fairly assuming that a vehicle on a city road will always encounter at least two street lamps on the road side, we select RF-based AoA measurements for the position estimate. Bearing measurements make localization possible with only two reference nodes, under certain conditions as discussed in the next section. For availability and scalability reasons, we choose to perform the localization process locally at the mobile node side. This requires that lamps act as reference nodes i.e periodically broadcast their position. Therefore, the mobile node, acting as a receiver, should be AoA capable and aware of its Orientation. RF-based AoA capability is commonly achieved by means of an antenna array [7].

\section{AOA TRIANGULATION BACKGROUND}

A node is said to be AoA capable if it is able to estimate the direction from which he received data from a neighbor. Such measurements, usually made by means of antenna arrays [7], are relative to the receiver's orientation. Orientation, defined as a fixed direction against which AoAs are measured, is represented in degrees in a clockwise direction from the North [9]. When the orientation is $0^{\circ}$ or pointing to the North, the AoA is absolute, otherwise, relative. In other words, the AoA capability provides the mobile node bearings to neighboring nodes with respect to its own axis. Triangulation is the process of determining the position of an object by using the bearings from known reference positions [11]. Fig. 2 illustrates the AoA triangulation principle with two reference neighbors:

- $\theta$ : Orientation of the mobile node $M$ (vehicle).

- $\tau_{1}$ (resp. $\tau_{2}$ ) : AoA or bearing of $M$ to $L_{1}$ (resp. $L_{2}$ ).

- $\phi_{12}=\tau_{2}-\tau_{1}$ : Visual angle ${ }^{2}$ between $L_{2}$ and $L_{1}$.

- $r_{1}$ (resp. $r_{2}$ ) : Distance between $M$ and $L_{1}$ (resp. $L_{2}$ ).

- $\left(X_{e}, Y_{e}\right)$ : The external coordinate system of the city map.

- $(X, Y)$ : The vehicle centered coordinate system.

In the absence of noise, the relation between the absolute bearings and the true location of the mobile node is:

$$
\tan \left(\alpha_{i}\right)=\frac{y-y_{i}}{x-x_{i}}, \text { with } \alpha_{i}=\theta+\tau_{i} \quad(i=1 . .2) .
$$

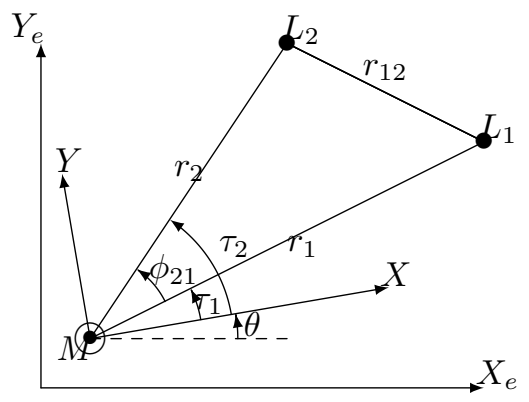

Fig. 2. Position Estimation using AoA Triangulation

\section{A. Triangulation with perfect data}

It is clearly shown in [14] [10] [9] that in order to estimate the position of the mobile node, at least two non-collinear reference neighbors are required. To estimate both position and orientation, at least three neighbors are required. In this work, we assume that the vehicle has an accurate estimate of its orientation by means of a digital compass from the INS for instance. Hence, we consider the triangulation case with two reference nodes. In this case, the intersection of bearing lines,

\footnotetext{
${ }^{2}$ Also called the bearing of $L_{2}$ relative to $L_{1}$
} 
in the reference frame $\left(X_{e}, Y_{e}\right)$, can be computed using the following equations [12]:

$$
\begin{aligned}
& x=x_{2}+\cos \left(\alpha_{2}\right) \frac{y_{2}-y_{1}-\tan \left(\alpha_{1}\right)\left(x_{2}-x_{1}\right)}{\cos \left(\alpha_{2}\right) \tan \left(\alpha_{1}\right)-\sin \left(\alpha_{1}\right)} \\
& y=y_{2}+\sin \left(\alpha_{2}\right) \frac{y_{2}-y_{1}-\tan \left(\alpha_{1}\right)\left(x_{2}-x_{1}\right)}{\cos \left(\alpha_{2}\right) \tan \left(\alpha_{1}\right)-\sin \left(\alpha_{1}\right)}
\end{aligned}
$$

where $\alpha_{i}=\theta+\tau_{i}$ is the absolute AoA from $L_{i}$, and $\left(x_{i}, y_{i}\right)$ are the coordinates of the reference nodes in the frame $\left(X_{e}, Y_{e}\right)$.

However, when the mobile node is directly between the two reference nodes, usually a third reference node is required to disambiguate. This leads to either solving a system of equations or optimization using Maximum Likelihood. In a city, this typically occurs in roundabouts or when the second neighbor is not on the same lane of the first one. To avoid the need of a third reference node, and to avoid complex computations, we propose to use the road knowledge to disambiguate the position estimation : the estimated position is simply the intersection if the current road segment with the segment $[L 1 L 2]$. Again, we observe the influence of the application context on the decision process.

For comparison, a Least Square (LS) closed form solution to the positioning problem with $\mathrm{N}$ reference nodes is:

$$
\hat{\mathbf{P}}=\left(\mathbf{G}^{T} \mathbf{G}\right)^{-1} \mathbf{G}^{T} \mathbf{h}
$$

where :

$\mathbf{G}=\left[\begin{array}{cc}\sin \left(\alpha_{1}\right) & -\cos \left(\alpha_{1}\right) \\ \vdots & \vdots \\ \sin \left(\alpha_{N}\right) & -\cos \left(\alpha_{N}\right)\end{array}\right], \mathbf{h}=\left[\begin{array}{c}x_{1} \sin \left(\alpha_{1}\right)-y_{1} \cos \left(\alpha_{1}\right) \\ \vdots \\ x_{N} \sin \left(\alpha_{N}\right)-y_{N} \cos \left(\alpha_{N}\right)\end{array}\right]$.

The LS algorithms assumes that the bearing measurement errors are relatively small, and the solution in Eq. (3) is obtained by solving the system of equations (written from Eq. (2)):

$$
x \sin \left(\alpha_{i}\right)-y \cos \left(\alpha_{i}\right)=x_{i} \sin \left(\alpha_{i}\right)-y_{i} \cos \left(\alpha_{i}\right)
$$

equivalent to:

$$
\mathbf{G P}=\mathbf{h} .
$$

For a quick overview of the analytic position estimation algorithms and their performance, the reader may refer to [15].

\section{B. Triangulation with noisy data}

In the presence of noise in the bearing measurements, it is important to study its effect on the position estimate. As seen in Eq. (5), the triangulation problem is equivalent to solving the system of equations constraining the position of the mobile node at the intersection of bearing lines. The problem can also be formulated as an observer process in the form $\vec{z}=h(\vec{x})$, where $\vec{x}=(x, y)$ is the mobile node position, and $\vec{z}$ denotes the observation vector relating the position to the bearing measurements. One way of formally quantifying the noise effect is the study of the determinant of the Jacobian of $\mathrm{h}\left(H=\frac{\delta h}{\delta x}\right)$, which is commonly referred to as the Geometric Dilution of Precision (GDOP). An intuitive and detailed derivation of the GDOP can be found in [16] for the case of two reference nodes. In the case of two reference nodes, the GDOP is given by:

$$
G D O P=\frac{r_{1} r_{2}}{\sin \left(\phi_{12}\right)} .
$$

Thus, the GDOP suggests that the position error tends to infinity when close to the line between the reference nodes ( $\phi_{12}$ tends to 0$)$. This also happens when the range product $\left(r_{1} r_{2}\right)$ becomes large. The best GDOP is obtained when the visual angle $\phi_{12}$ is a right angle.

\section{NETWORK MODEL}

The considered network is a Wireless Sensor Network (WSN) where we distinguish two types of nodes : reference nodes and mobile node(s). Reference nodes are attached to the street lamps, and they periodically transmit a beacon containing their position with a period $T_{\text {beacon }}$. Mobile nodes are attached to the vehicles, and are AoA capable.

The topology of the network is dictated by the spatial distribution of the lamps in the city, whereas the position of the mobile nodes changes with time along the road. The network is supposed to operate with the same communication range $R_{\max }$ for all the nodes. In order to ensure the connectivity of the network $R_{\max }$ should be more or equal than the maximum inter-Lamp spacing $S$ :

$$
R_{\max } \geq S \text {. }
$$

We assume that $R_{\max }=S$, which is a fair trade-off to minimize the effect of collisions at medium access level.

At MAC layer, the nodes use unslotted CSMA/CA algorithm to access to the medium according to the IEEE 802.15.4 standard [17]. Finally, the physical layer is assumed to be perfect i.e the radio coverage of a transmitting node is circular with radius $R_{\max }$.

\section{Mobility Model}

In order to simulate the mobility of the mobile node, we adopt a simple mobility model involving a trajectory on a route and constant speed (see Fig. 3(a)). A trajectory is given by a set of waypoints $\left\{W_{1} \ldots W_{n}\right\}$ where each waypoint pair forms a segment. Each segment has a direction $\theta_{j}=\tan ^{-1} \frac{y_{j+1}-y_{j}}{x_{j+1}-x_{j}}$. The number of waypoints depends on the route geometry. For example a new waypoint is required at every direction change in the route. In order to travel along a given trajectory $W$ at a constant speed $v$, we propose a simple recursive mobility model in Fig. 3(b). At every sampling period $T_{s}$, the position of the mobile node is updated with a displacement $d s=v \times$ $T s$.

\section{Vi. Position Estimation Algorithm}

When the mobile node receives a beacon packet from a reference node, it retrieves the sender's position $\left(x_{i}, y_{i}\right)$ from the received packet and estimate its absolute bearing to it $\left(\alpha_{i}\right)$, then adds a time-stamp $\left(t_{1}\right)$ according to its internal clock. It then waits for a packet reception from a second node. Once received after a fixed maximum timeout $\left(T_{s}\right)$, it estimates its position by triangulation according to Eq. (1), and then checks 


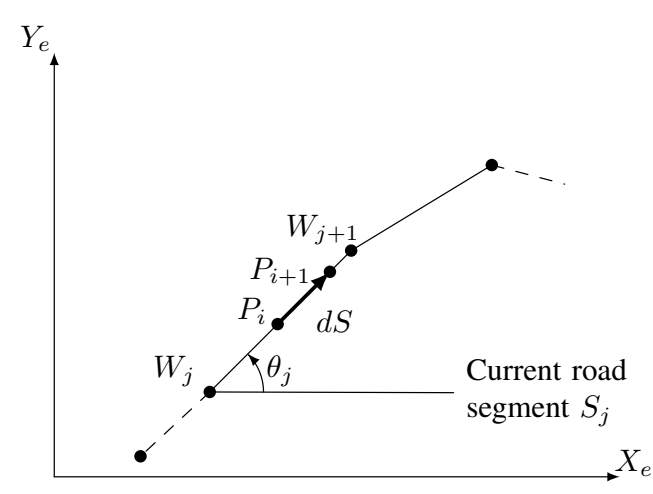

(a) Waypoint Mobility Model

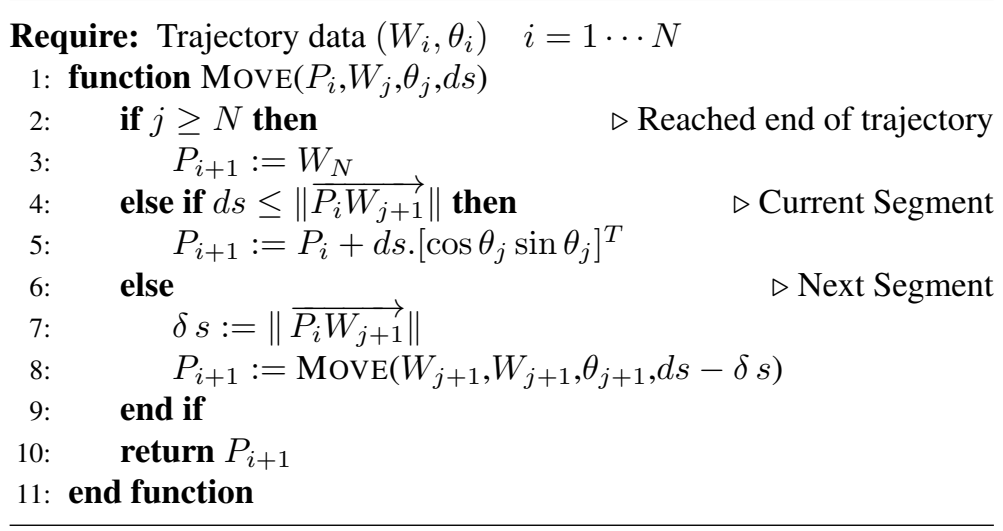

(b) Waypoint Mobility Algorithm

Fig. 3. Waypoint Mobility

position consistency against GDOP using the knowledge of the maximum communication range $R_{\max }$ of the network.

Note that checking the inter-packet arrival time between the two beacons is very important when working with a realistic MAC. Due to collisions, the second reference node may be observed on a different position. In this case, the oldest observed node is dropped. Finally, when a beacon is received from an already observed node, the internal structure $L_{i}$ is updated with time and bearing information.

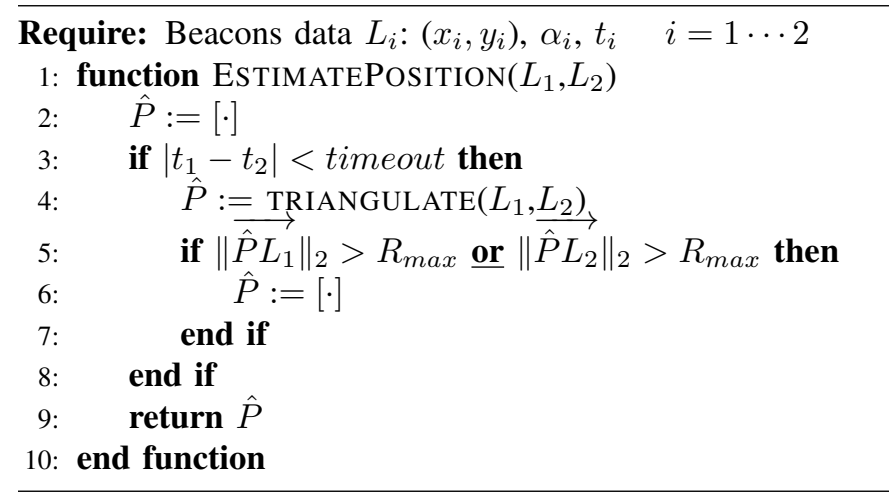

Fig. 4. Position Estimation Algorithm

\section{Simulation Results}

In order to reflect the smart city topology, we adopted the following simulation methodology.

1) Select a road of interest in a city using Google Maps Engine.

2) Detect street lamps and add markers on each. Each marker will get the lamp coordinates (Longitute, Latitude).

3) Draw a trajectory along the same road. Each trajectory waypoint will have its coordinates.

4) Export the created map to a KML file, say scenario. $k m l$.
5) Parse the exported KML file and extract the coordinates of the lamps and the trajectory.

6) Project those coordinates using the Universal Transverse Mercator (UTM) projection and shift the coordinates to center the map.

7) Generate the coordinates from previous step into separate data files to feed the WSN simulator.

The resulting network and trajectory is depicted in the Fig. 5. We choose this trajectory as it contains a roundabout and exhibits GDOP (ex. in the roundabout and when crossing between lamps 5 and 7).

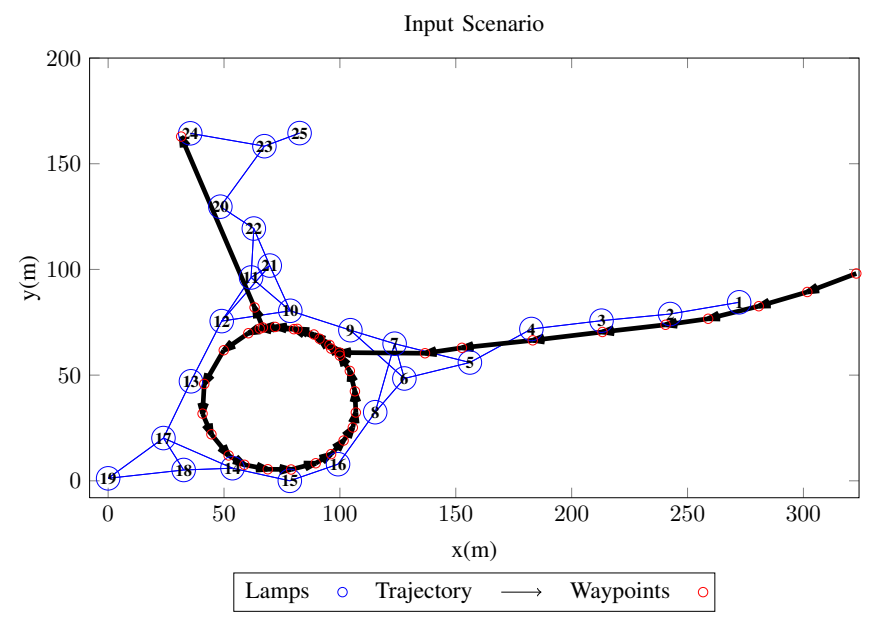

Fig. 5. Extracted navigation scenario

For WSN simulation, we used the WSNET simulator [18]. WSNET is a modular discrete event simulator that is able of simulating multihop wireless networks, in particular WSN. It is programmed in $\mathrm{C}$, which eases porting the code to real hardware. It provides realistic MAC and PHY layers and supports mobility. We implemented a mobility model according to Section $\mathrm{V}$ and two application modules for reference fixed nodes and one mobile node. The mobile node estimated his position upon each beacon reception according 
to Section VI. The sampling period of the mobility model $T_{s}$ was fixed to $200 \mathrm{~ms}$.

The simulation strategy is depicted by Fig. 6 . The beacon transmission period was fixed to $1 s$, which constrains the expected position update rate to $1 \mathrm{~s}$. The vehicle speed $v$ was fixed to $70 \mathrm{~km} / \mathrm{h}$, the maximum speed limit. With this configuration, we study the effect of collisions at MAC layer and the bearing noise $\sigma_{A o A}$ on the positioning performance in terms of: position update rate and accuracy. The bearing noise was generated by adding to the true bearing a normal Gaussian noise with standard deviation $\sigma_{A o A}$. Finally, the maximum communication range was set as discussed in Section IV to $R_{\max }=S=35 \mathrm{~m}$, which is the maximum inter-lamp spacing observed in the scenario in Fig. 5.

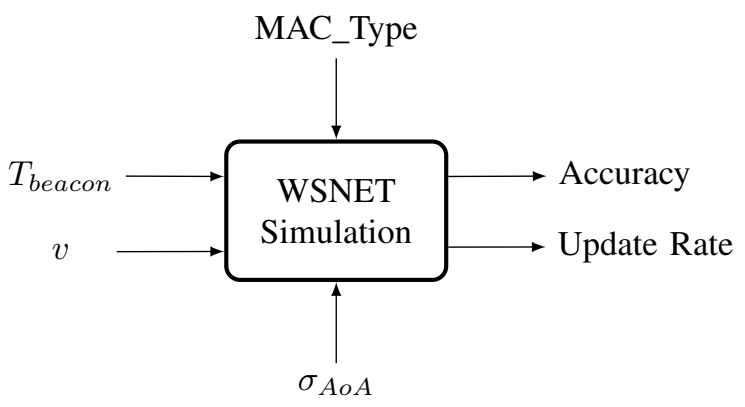

Fig. 6. Simulation Parameters - Positioning performance as output

\section{A. The effect of collisions (MAC Layer)}

When reference nodes transmit beacons, collisions may occur, thus preventing the mobile node from estimating its position despite of its physical presence in the vicinity of the lamps. As a consequence, the position update rate is affected. To evaluate this impact, we compared the case of a perfect MAC layer with a realistic one i.e implementing IEEE 802.15.4 unslotted CSMA/CA with the default standard parameters ${ }^{3}$. We ran 500 simulations, with $\sigma_{A o A}=0$, and analyzed the average position update rate. Fig. 7 shows the resulting position update rate distribution. This result suggests that, even on a relatively short trajectory as in Fig. 5, beacon packet miss degrades the packet reception rate up to $7 \mathrm{~s}$ in presence of collisions. This means, that for a vehicle traveling at $70 \mathrm{~km} / \mathrm{h}$, there will be no position update for approx. 14 meters. Assessing the worst position update rate is very important when implementing a hybrid scheme with Dead Reckoning (Fig. 1).

\section{B. The effect of AoA measurement noise}

Noisy AoA measurements lead to positioning errors that may become very large due to GDOP. In order to separately study the effect of noise measurements on positioning errors, we run simulations with a perfect MAC layer.

\footnotetext{
${ }^{3}$ max-csma-backoff=4,min-backoff-exponent=3, max-backoff-exponent $=5$
}

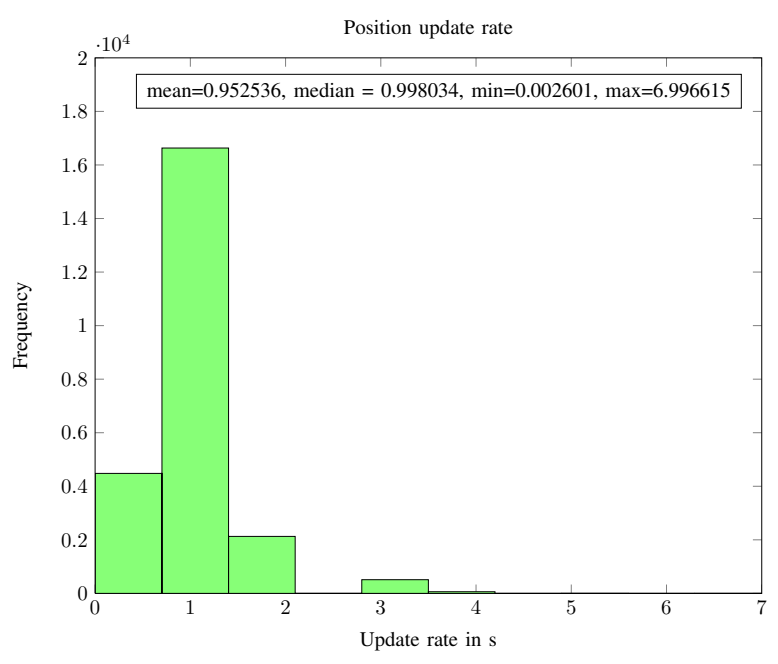

Fig. 7. Effect of collisions on position update rate.

1) Effect of GDOP: In order to show the effect of the GDOP, we ran two simulations under bearing noise of $1^{\circ}$ : with GDOP mitigation disabled, and then enabled. The results are depicted in Fig. 8. From Fig. 8(a), we observe that position samples 70 and 160 lead to extremely high errors due to GDOP, and when the GDOP is activated, such estimates are dropped and come to lowering the average estimation error (Fig. 8(b)).

2) Performance of Positioning Algorithm: The Root Mean Square Error (RMSE) for the position estimation algorithm is computed by averaging 500 independent simulations:

$$
R M S E=\sqrt{\frac{1}{n} \sum_{i=1}^{n}\left\|\hat{P}_{i}-P_{i}\right\|_{2}^{2}}
$$

where $n$ is the number of successful position estimation samples. Fig. 9 shows the algorithm performance versus the standard deviation of the bearing measurement noise. As

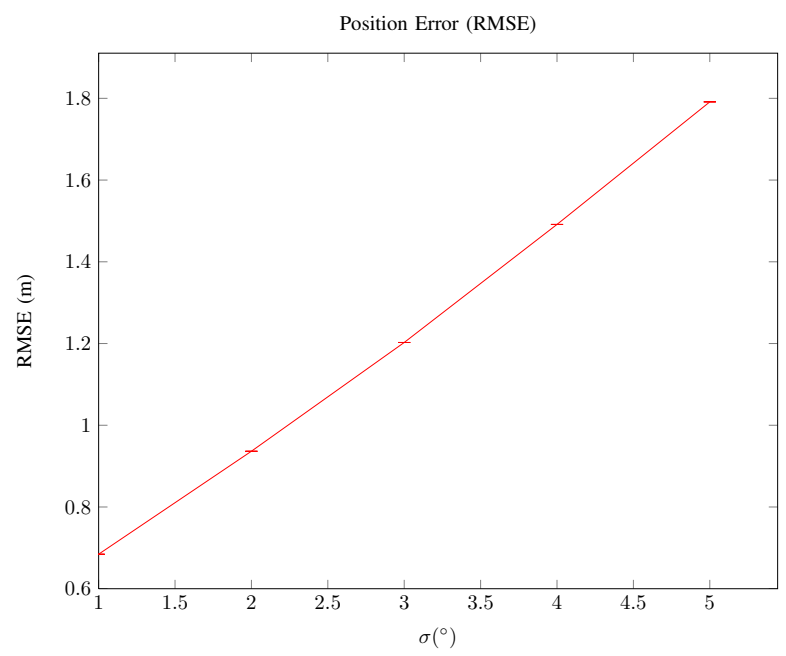

Fig. 9. The RMSE performance of the proposed algorithm. 


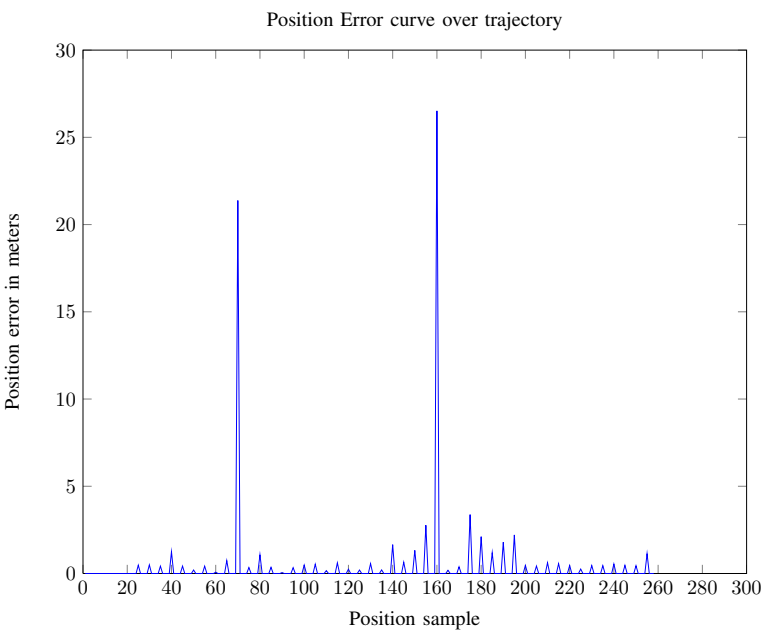

(a) Without GDOP mitigation

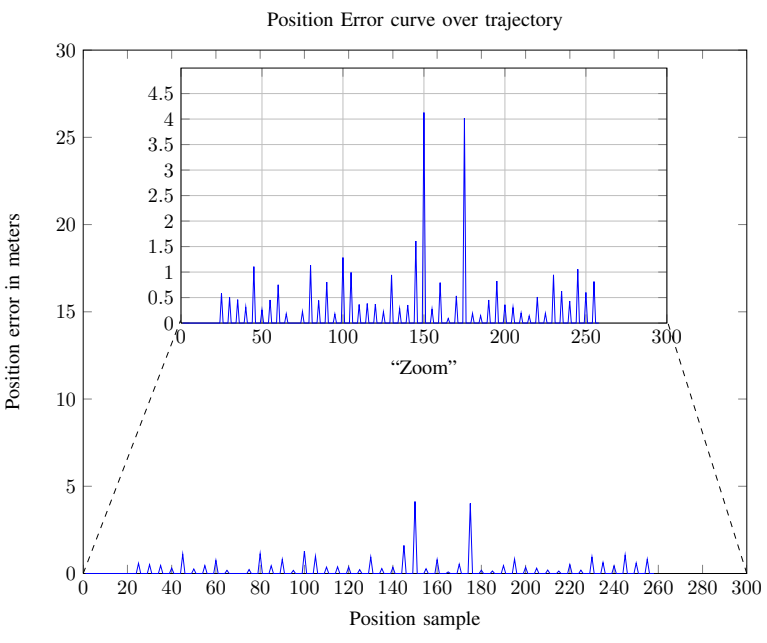

(b) With GDOP mitigation

Fig. 8. GDOP effect

expected, the positioning error increases with the standard deviation of the bearing measurements noise. Note that, if we plot the same curve without GDOP mitigation, the RMSE analysis would be made impossible due to the effect the extremely high errors.

\section{CONCLUSION}

In this paper, we studied the performance of triangulation using AoA in a realistic smart urban environment that exhibits topology related constraints. We proposed a simple method to cope with dilution of precision. Simulations results show that distribution of the anchor nodes highly affected both positioning accuracy (dilution of precision) and beacon packets reception rate (collisions). Therefore, AoA triangulation alone is not sufficient, and a hybrid navigation solution is required as suggested in Section II. Future fork will concern the implementation of the Dead Reckoning block and its integration with the AoA-based triangulation feature.

\section{ACKNOWLEDGMENT}

This work is partially supported by a grant from CPER Nord-Pas-de- Calais/FEDER Campus Intelligence Ambiante, the FP7 SMART CITIES VITAL, the Etipops i-Lab and the ANRT (Association Nationale de la Recherche Technique) through the CIFRE grant 2012/0860.

\section{REFERENCES}

[1] A. Alkandari, M. Alnasheet, and I. Alshaikhli, "Smart cities: Survey," Journal of Advanced Computer Science and Technology Research, vol. 2, no. 2, pp. 79-90, 2012.

[2] T. Watteyne and K. S. J. Pister, "Smarter cities through standards-based wireless sensor networks," IBM Journal of Research and Development, vol. 55 , no. $1 \& 2$, pp. 80-89, 2011.

[3] I. F. Akyildiz, W. Su, Y. Sankarasubramaniam, and E. Cayirci, "Wireless sensor networks: a survey," Computer Networks, vol. 38, no. 4, pp. 393 422, 2002.

[4] G. W. Denardin, C. H. Barriquello, A. Campos, R. A. Pinto, M. A. Dalla Costa, and R. N. do Prado, "Control network for modern street lighting systems," in IEEE International Symposium on Industrial Electronics (ISIE), Gdańsk - Poland, June 2011, pp. 1282-1289.
[5] F. Domingo-Perez, A. Gil-de Castro, J. Flores-Arias, F. BellidoOuteirino, and A. Moreno-Munoz, "Lighting control system based on dali and wireless sensor networks," in The third IEEE PES Innovative Smart Grid Technologies (ISGT), Jan 2012, pp. 1-6.

[6] Y. Liu, Z. Yang, X. Wang, and L. Jian, "Location, localization, and localizability," Journal of Computer Science and Technology, vol. 25, no. 2, pp. 274-297, 2010

[7] G. Mao, B. Fidan, and B. Anderson, "Wireless sensor network localization techniques," Computer Networks, vol. 51, no. 10, pp. 2529-2553, 2007.

[8] D. Niculescu and B. Nath, "Ad hoc positioning system (aps) using aoa," in The 22nd Annual Joint Conference of the IEEE Computer and Communications Societies (INFOCOM), vol. 3, 30 March - 3 April 2003, pp. 1734-1743.

[9] P. Rong and M. L. Sichitiu, "Angle of arrival localization for wireless sensor networks," in Sensor and Ad Hoc Communications and Networks, 2006. SECON'06. 2006 3rd Annual IEEE Communications Society on, vol. 1, 2006, pp. 374-382.

[10] M. Betke and L. Gurvits, "Mobile robot localization using landmarks," IEEE Transactions on Robotics and Automation, vol. 13, no. 2, pp. 251263, 1997

[11] J. S. Esteves, A. Carvalho, and C. Couto, "Generalized geometric triangulation algorithm for mobile robot absolute self-localization," in IEEE International Symposium on Industrial Electronics (ISIE)), vol. 1, June 2003, pp. 346-351.

[12] I. Amundson, X. Koutsoukos, J. Sallai, and A. Ledeczi, "Mobile sensor navigation using rapid rf-based angle of arrival localization," in The 17th IEEE Real-Time and Embedded Technology and Applications Symposium (RTAS), April 2011, pp. 316-325.

[13] A. Boukerche, H. A. Oliveira, E. F. Nakamura, and A. A. Loureiro, "Vehicular ad hoc networks: A new challenge for localization-based systems," Computer Communications, vol. 31, no. 12, pp. 2838-2849, 2008.

[14] C. D. Mcgillem and T. S. Rappaport, "A beacon navigation method for autonomous vehicles," IEEE Transactions on Vehicular Technology, vol. 38, no. 3, pp. 132-139, 1989.

[15] R. M. Vaghefi, M. R. Gholami, and E. Strom, "Bearing-only target localization with uncertainties in observer position," in The 21st IEEE International Symposium on Personal, Indoor and Mobile Radio Communications Workshops (PIMRC Workshops), September 2010, pp. 238242.

[16] A. Kelly, "Precision dilution in triangulation based mobile robot position estimation," in Intelligent Autonomous Systems, 2003.

[17] “IEEE 802.15.4 MAC standard, 2006 revision," http://www.ieee.org/, 2008.

[18] G. Chelius, A. Fraboulet, and E. Benhamida, "WSNET: a modular eventdriven wireless network simulator," http://wsnet.gforge.inria.fr/, 2006. 